\title{
Approval may embolden industry to combine cancer therapies
}

In the fight against cancer, researchers agree that it is a good idea to combine targeted drugs with each other or with immunotherapies: tumor cells mutate and can become resistant to treatment that works via one route, so an additional therapy makes effective treatment more likely. But for drug companies, deciding to test promising combinations has not been easy.

In some cases, pharmaceutical developers simply view it as against their interests to collaborate. "Drug companies too often are reluctant to work together, because they think it won't be to their advantage," observes Carlos Moreno of the Winship Cancer Institute of Emory University in Atlanta.

For example, one company might have a candidate therapy that would make sense to test with a drug from a different firm. But because the two firms hold the patents to each separately, both parties might worry about future liabilities, intellectual property (IP) rights, and secondary IP (that is, IP issues that might arise from unexpected new therapeutic benefits from combining the drugs).

Fears such as this might make working together seem too costly to individual firms. However, it can be "a perception problem" as much as a real one, says David Bonk, a consultant pharmaceuticals patent lawyer, which can stall discussions before further research or trials even get off the ground.

\section{Moving forward}

Over the last year, however, researchers and industry watchers have become hopeful that a turning point has been reached that will make future collaborations more likely. In January of 2014, the US Food and Drug Administration (FDA) approved Tafinlar (dabrafenib) and Mekinist (trametinib), two drugs from GlaxoSmithKline, as the first duo of targeted agents to be used in combination for treating melanoma. Each had previously been approved individually for patients with particular mutations in BRAF, a gene that encodes the enzyme B-Raf: Tafinlar blocks one part of the pathway and Mekinist another. In trials, patients responded to treatment for a longer period of time when receiving the two together than when taking Tafinar alone.

In September, Roche announced promising phase 3 results for the combination of two investigational drugs:

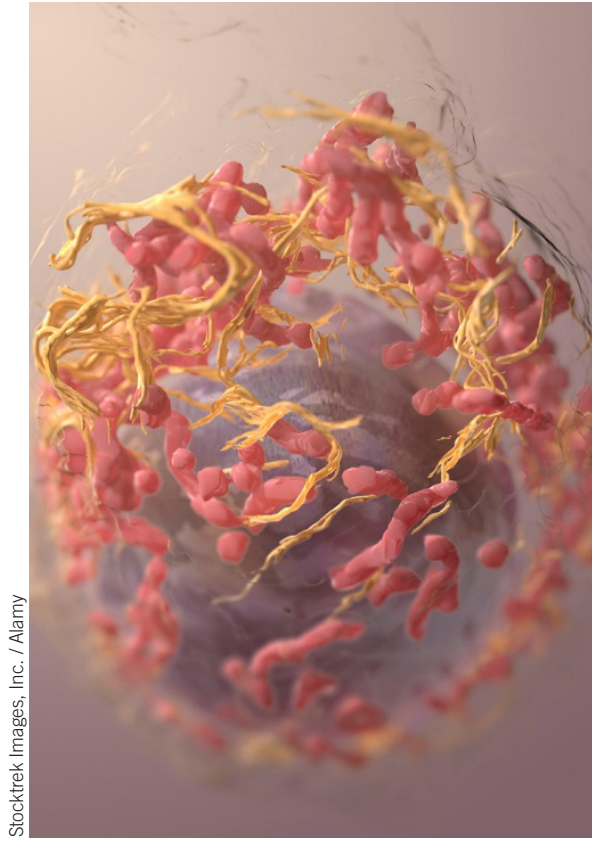

It takes two: Combo treats melanoma.

cobimetinib, a mitogen-activated protein kinase (MEK1) inhibitor co-developed by the Roche subsidiary Genentech and the South San Francisco-based Exelixis, with vemurafenib, a melanoma drug targeting B-Raf. Three months later, Roche applied for FDA approval to give the drugs as a combined treatment for melanoma.

Also in December 2014, Bristol-Myers Squibb announced that it would begin a phase 1 trial to test Opdivo (nivolumab), its inhibitor of the PD-1 immune checkpoint, with mogamulizumab, a CCR4-specific antibody from Kyowa Hakko Kirin, a Japanese firm. Meanwhile, a phase 1 trial to treat breast cancer and ovarian cancer is underway using a combination of AstraZeneca's drug olaparib and either BKM120 or BYL719 from Novartis, another pharmaceutical giant.

Public and private partners are playing bigger collaborative roles, too. In 2013, GSK formed a consortium with cancer centers to develop combination therapies, for example. Meanwhile, the Melanoma Research Alliance and the Prostate Cancer Foundation, the biggest private philanthropic organizations in each respective field, have both made funding combination therapy research a goal.

The US National Cancer Institute (NCI) plans to launch a database of drug combinations this spring, to be named the
NCI Almanac. The information it provides will go some way in helping to figure out which drugs might work better when given together than when given individually.

The database will pool results from an ambitious project the NCI began a few years ago that helps rationalize drug combination approaches. Every combination of FDAapproved oncology drugs was tested against cancer cell lines the NCI has characterized, to create a matrix that plots the efficacy of each combination. These data will become available as a web tool for drug developers to see which drug pairs are active against which cell lines, and which might potentially be toxic.

\section{Avoiding toxicity}

"Drug companies are developing targeted cancer agents faster than they're able to develop appropriate models for figuring out how to combine them," says James Doroshow, deputy director for clinical and translational research at the NCI and one of the developers of the database. "There are simply too many possibilities, and they don't have the resources to screen them."

Putting drugs together can introduce unexpected problems not seen when using an agent alone. "Toxicity is a big issue," says Doroshow, and when it shows up in trials, it adds to the expense of a developing combination therapy. A phase 1 trial that combined ipilimumab, an immunotherapeutic checkpoint inhibitor from Bristol-Myers Squibb, and vemurafenib was stopped in April 2013 after subjects showed signs of liver toxicity.

Beyond its research projects, the NCI also provides legal frameworks for combination trials done by multiple parties. These agreements lay out IP conditions for doing trials with the NCI, establishing a standard that avoids complications.

In the end, the science will make it clear that collaborations are economically beneficial for companies, according to Bonk. "Unlike older chemotherapy, companies know which patients will respond to a targeted drug or immunotherapy they're developing," he says. "Working out how to combine them, even with someone else's drug, might reveal new subpopulations that would benefit, and open up new avenues."

"In my experience, if the parties are incentivized, the legal questions become easy to sort out," he adds.

Boer Deng 